\title{
ON THE EVOLUTION OF PROTO-PLANETARY NEBULAE
}

\author{
J.Y. HU and B.W. JIANG \\ Beijing Observatory, Academia Sinica, Beijing, 100080, China \\ and \\ S. SLIJKHUIS \\ Astronomical Institute, University of Amsterdam, The Netherlands
}

The ages of PNNe in our sample (Hu et al., 1990) were derived from the inner radius of the dust shell using the radiative transfer model fitting with SED and expansion velocities of envelopes obtained from radio molecular lines observations (if without radio data a $\mathrm{V}_{\text {exp }}$ of $15 \mathrm{~km} / \mathrm{sec}$ was assumed). The objects are classified into the following groups:

1. invisible;

2. M-type;

3. F (or G)-type; and

4. $\mathrm{F}$ (or $\mathrm{G}$ )-type with $\mathrm{H}_{\alpha}$ emission.

The average ages of different groups are 97, 171, 247 and 477 years respectively. We considered that it is an evolutionary sequency.

The luminosities of the objects in the sample in which $\mathrm{CO}$ or $\mathrm{OH}$ maser lines were detected were derived using kinematic distances and total flux densities. The luminosities are relative to the IRAS color both for C-rich and O-rich objects. More luminous objects have a larger $\log (\mathrm{S} 60 / \mathrm{S} 25)$. When an AGB star evolves into the PPN phase, it leaves the AGB evolution track and turns to the right on the IRAS color-color diagram $(\log \mathrm{S} 25 / \mathrm{S} 12$ vis $\log \mathrm{S} 60 / \mathrm{S} 25)$. The model calculation (Slijkhuis et al., 1992) shows that in the early stages the objects evolve to the right almost parallel with the $\mathrm{X}$-axis. Based on the relations of luminosity and logS60/S25 we considered that the turn-off points from the AGB evolution track on the IRAS color-color diagram to the PPN phase are relative to the luminosities i.e. the initial mass. Massive stars turn off in the upper part of AGB evolution track and low mass stars turn off in the lower part.

\section{References}

Hu J.Y., de Jong T., Slijkhuis S., 1990, 'From Miras to Planetary Nebulae', 487-489. Slijkhuis S., 1992, Ph.D. thesis, Chapter 6, Univ. of Amsterdam 